\title{
Undifferentiated Pleomorphic Sarcoma with Neoplastic Fever: A Retrospective Study
}

\author{
Jiaqiang Wang (D) \\ Shuping Dong' \\ Jianpo Zhang ${ }^{2}$ \\ Shilei Gao' \\ Zhehuang $\mathrm{Li}^{\prime}$ \\ Po Li ${ }^{1}$ \\ Junhui Yuan ${ }^{3}$ \\ Zhichao Tian (D) \\ 'Department of Bone and Soft Tissue, \\ the Affiliated Cancer Hospital of \\ Zhengzhou University and Henan Cancer \\ Hospital, Zhengzhou, Henan Province, \\ 450008, People's Republic of China; \\ 2Pathology Department, the Affiliated \\ Cancer Hospital of Zhengzhou University \\ and Henan Cancer Hospital, Zhengzhou, \\ Henan Province, 450008, People's \\ Republic of China; ${ }^{3}$ Medical Imaging \\ Department, the Affiliated Cancer \\ Hospital of Zhengzhou University and \\ Henan Cancer Hospital, Zhengzhou, \\ Henan Province, 450008, People's \\ Republic of China
}

Correspondence: Zhichao Tian Department of Bone and Soft Tissue, the Affiliated Cancer Hospital of Zhengzhou University and Henan Cancer Hospital, Dongming Road, Zhengzhou, Henan Province, 450008, People's Republic of China

Tel +86- |35924|373|

Fax +86-37I-6596I505

Email tianzhichaoyy@163.com
Background: Although the annual incidence of undifferentiated pleomorphic sarcoma (UPS) is extremely low, it can be subdivided into different subtypes. UPS with fever of unknown origin (also known as neoplastic fever) is a specific subtype of UPS, which shows certain clinical features that differentiate it from other UPS subtypes. However, no studies have focused on this rare UPS subtype. This study retrospectively analyzed the clinical data of patients with UPS to provide a reference for the diagnosis and treatment of UPS with neoplastic fever.

Methods: This study included patients with UPS who were diagnosed and treated between June 2012 and June 2018. We examined whether these patients had a history of neoplastic fever. The characteristics of patients with UPS with neoplastic fever were summarized and analyzed.

Results: We reviewed the medical records of 183 patients with UPS. Seven $(3.83 \%)$ of these patients had neoplastic fever. In patients with UPS with neoplastic fever, the primary lesions were located in the extremities and across the muscle space. In these patients, magnetic resonance imaging showed necrosis within the tumor body and extensive soft tissue edema around the tumor body. Patients with UPS with neoplastic fever had a lower metastasis rate $(14.29 \%$ vs $44.94 \%)$ and a higher 3-year survival rate $(85.71 \%$ vs $59.55 \%)$ than those without neoplastic fever.

Conclusion: UPS with neoplastic fever is characterized by intratumoral necrosis and extensive edema of the surrounding soft tissues. Patients with UPS with neoplastic fever may have a better prognosis than those without neoplastic fever.

Keywords: undifferentiated pleomorphic sarcoma, neoplastic fever, fever of unknown origin, sarcoma

\section{Introduction}

Soft tissue sarcomas (STSs) are rare malignant tumors of mesenchymal origin, comprising approximately 50 subtypes. ${ }^{1}$ Some types of STS lack defined characteristics for a specific sarcoma subtype; their cells are less differentiated, and reversion to a state that contains a population of varying morphological cell types (such as spindle cells, histiocytes, and giant cells) is noted. ${ }^{2}$ This type of tumor is known as undifferentiated pleomorphic sarcoma and was referred to as malignant fibrous histiocytoma until 2002. ${ }^{3}$ Its incidence in the population is extremely low, with only approximately $1.5 \%$ new cases per 1 million people per year. ${ }^{4}$ At present, there are few clinical reports specifically targeting UPS. Most data on the treatment and prognosis of UPS have been obtained from clinical trials on STS. ${ }^{5,6}$ Currently, the main treatments for early UPS are surgery and 
radiotherapy. ${ }^{6,7}$ Neoadjuvant therapy is also required for high-risk early UPS. ${ }^{8,9}$ The 5 -year survival rate for early UPS is less than $50 \% .{ }^{10}$ Systemic treatment of advanced UPS includes chemotherapy, targeted therapy, and immunotherapy. ${ }^{7,11}$ The median overall survival for latestage UPS is approximately 1 year. ${ }^{6,11}$

Although the annual incidence of UPS is extremely low, it can be subdivided into different subtypes. ${ }^{12}$ UPS with fever of unknown origin (also known as neoplastic fever) is a specific subtype of UPS. This UPS subtype can develop anywhere in the body, and its incidence rate is even lower than the normal subtypes. ${ }^{13-18}$ UPS with neoplastic fever has some clinical features which differentiates it from other UPS subtypes. However, no studies have focused on this rare UPS subtype. Our hospital is a major center for the diagnosis and treatment of sarcoma in a province with a population of 100 million in Central China; ${ }^{19,20}$ thus, we have accumulated data from a large number of patients with UPS, including those with UPS with neoplastic fever. In this study, we retrospectively analyzed the clinical data of patients with UPS to provide a reference for the diagnosis and treatment of UPS with neoplastic fever.

\section{Methods}

This study aimed to retrospectively analyze the clinical data of patients with UPS, to provide details on the diagnosis and treatment of UPS with neoplastic fever. After obtaining ethical approval from the Affiliated Cancer Hospital of Zhengzhou University, we reviewed the medical records of all patients with UPS treated at the Affiliated Cancer Hospital of Zhengzhou University between June 2012 and August 2018. The inclusion criteria were as follows: 1) pathologically confirmed UPS and 2) complete data regarding medical records, imaging studies, and follow-up records.

We examined whether the included patients had a history of neoplastic fever. Neoplastic fever was defined as fever of unknown origin, after all potential causes were ruled out. We also analyzed the following variables: age, Eastern Cooperative Oncology Group performance status (ECOG PS) score at diagnosis, location and size of the primary tumor, tumor stage at initial diagnosis, magnetic resonance imaging (MRI) findings at the onset of neoplastic fever, biopsy or postoperative pathology findings, adjuvant therapy, recurrence, metastasis, and 3-year survival rate.

\section{Statistical Analysis}

Quantitative variables are presented as the median (range) or number of patients (percentage). The recurrence rate was defined as the percentage of patients who showed relapse after the first surgery. The metastasis rate was defined as the percentage of patients who developed distant metastases after the initial diagnosis. The 3-year survival rate was defined as the percentage of patients who survived for 3 years after surgery.

\section{Results}

We reviewed the medical records of 183 patients with UPS; seven of these patients had neoplastic fever. Thus, the incidence of neoplastic fever was $3.83 \%$ in patients with UPS.

\section{Characteristics of Patients with UPS with Neoplastic Fever}

Detailed information of the seven patients with UPS with neoplastic fever is shown in Table 1. Of the seven patients, four were female and three were male; the mean age was $62 \pm 8.08$ years; and the ECOG PS score was 0 or 1 . In all patients, the primary lesions were located in the extremities and intermuscular space. The primary lesions were all relatively large at the first diagnosis, ranging from $4.8 \mathrm{~cm}$ to $18.0 \mathrm{~cm}$. Neoadjuvant chemotherapy was attempted in four patients, but they could not complete the treatment owing to fever; one patient could not complete neoadjuvant radiotherapy owing to fever. Radiotherapy and chemotherapy did not relieve neoplastic fever in these patients. All patients underwent extensive resection of the primary tumor. Fever symptoms disappeared in all patients after surgery. Four patients received adjuvant radiotherapy postoperatively, and two of them experienced recurrence. Of the seven patients, four experienced at least one postoperative recurrence; one patient developed extensive pulmonary metastasis and died 2.5 years after the initial diagnosis. Six patients were still alive 3 years after surgery.

\section{Comparison Between Patients with UPS with and without Neoplastic Fever}

To further investigate the clinical characteristics of patients with UPS with neoplastic fever (defined as the UPS with neoplastic fever group), we compared data between patients with UPS with and without neoplastic fever. A total of 89 patients were included in the UPS without 
Table I Clinical Characteristics of Patients with UPS with Neoplastic Fever

\begin{tabular}{|l|c|c|c|c|c|c|c|c|c|c|}
\hline $\begin{array}{l}\text { Patient } \\
\text { No. }\end{array}$ & Sex & $\begin{array}{c}\text { Age } \\
\text { (Years) }\end{array}$ & $\begin{array}{c}\text { ECOG } \\
\text { PS }\end{array}$ & $\begin{array}{c}\text { Primary } \\
\text { Site }\end{array}$ & $\begin{array}{c}\text { TNM } \\
\text { Stage }\end{array}$ & $\begin{array}{c}\text { Primary } \\
\text { Size (cm) }\end{array}$ & $\begin{array}{c}\text { Neoadjuvant } \\
\text { Therapy }\end{array}$ & Recurrence & Metastasis & $\begin{array}{c}\text { 3-Year } \\
\text { Survival }\end{array}$ \\
\hline I & Female & 68 & 0 & Thigh & III & 9.8 & Unfinished & No & No & Yes \\
2 & Male & 54 & I & Thigh & III & 12.8 & Unfinished & Yes & No & Yes \\
3 & Male & 65 & 0 & Thigh & III & 18.0 & Unfinished & Yes & No & Yes \\
4 & Male & 57 & I & Lower leg & III & 9.6 & Unfinished & No & No & Yes \\
5 & Female & 66 & 0 & Upper arm & III & 7.2 & NA & Yes & No & Yes \\
6 & Female & 73 & 0 & Upper arm & II & 4.8 & NA & No & No & Yes \\
7 & Male & $5 \mathrm{I}$ & 0 & Thigh & III & 15.0 & Unfinished & Yes & Yes & No \\
\hline
\end{tabular}

Abbreviations: UPS, undifferentiated pleomorphic sarcoma; ECOG PS, Eastern Cooperative Oncology Group performance status; NA, not applicable.

neoplastic fever group. The site and size of the primary tumor and tumor stage at the initial diagnosis were between the two groups. Basic information for the two groups is presented in Table 2.

Pathological manifestations were similar between patients with UPS with and without neoplastic fever (Figure 1). Histopathologically, all cases of UPS showed high cellularity, myxoid area $<10 \%$, and pleomorphic nuclear atypia. In the UPS with neoplastic fever group, MRI showed necrosis inside the tumor body. All tumors involved the muscular space. Extensive soft tissue edema was observed around the tumor (Figure 2). These features were not noted in most cases in the UPS without neoplastic fever group (Figure 2).

The recurrence rate was similar between patients with UPS with and without neoplastic fever $(57.14 \%$ vs 53.93\%). In contrast, the UPS with neoplastic fever group showed a lower metastasis rate $(14.29 \%$ vs $44.94 \%)$ and a higher 3 -year survival rate $(85.71 \%$ vs $59.55 \%$ ) than the UPS without neoplastic fever group. Owing to the small number of cases in the UPS with neoplastic fever group and the large difference in the number of cases between the two groups, the statistical error was too large. Therefore, we did not perform a statistical comparison of the differences in the data between the two groups.

\section{Discussion}

In this retrospective study, we found that 7 (3.83\%) of the 183 patients with UPS had neoplastic fever. In these patients, the primary tumors were located in the extremities and muscle space. On MRI, these patients showed necrosis within the tumor body and extensive soft tissue edema around the tumor body. Patients with UPS with neoplastic fever had a lower metastasis rate and a higher
Table 2 Comparison of Clinical Characteristics Between Patients with UPS with and without Neoplastic Fever

\begin{tabular}{|c|c|c|}
\hline Characteristics & $\begin{array}{l}\text { Fever UPS } \\
\text { Group }(n=7)\end{array}$ & $\begin{array}{l}\text { Normal UPS } \\
\text { Group }(n=89)\end{array}$ \\
\hline \multicolumn{3}{|l|}{ Gender } \\
\hline Male & 4 & 49 \\
\hline Female & 3 & 40 \\
\hline Age (years) & $62 \pm 8.08$ & $59 \pm 10.12$ \\
\hline \multicolumn{3}{|l|}{ ECOG PS } \\
\hline 0 & 5 & 76 \\
\hline 1 & 2 & 13 \\
\hline \multicolumn{3}{|l|}{ Primary site } \\
\hline Upper arm & 2 & 26 \\
\hline Thigh & 4 & 54 \\
\hline Lower leg & 1 & 9 \\
\hline Primary tumour size $(\mathrm{cm})$ & $11.02 \pm 4.56$ & $7.28 \pm 6.83$ \\
\hline \multicolumn{3}{|l|}{ Stage } \\
\hline II & 1 & 18 \\
\hline III & 6 & 71 \\
\hline \multicolumn{3}{|l|}{$\begin{array}{l}\text { Peritumor soft tissue edema } \\
\text { and tumor necrosis (MR) }\end{array}$} \\
\hline Yes & 7 & 21 \\
\hline No & 0 & 68 \\
\hline \multicolumn{3}{|l|}{ Postoperative adjuvant therapy } \\
\hline No & 3 & 24 \\
\hline Yes & 4 & 65 \\
\hline Recurrence rate & $57.14 \%(4 / 7)$ & $53.93 \%(48 / 89)$ \\
\hline Metastasis rate & $14.29 \%(1 / 7)$ & $44.94 \%(40 / 89)$ \\
\hline 3-year survival rate & $85.71 \%(6 / 7)$ & $59.55 \%(53 / 89)$ \\
\hline
\end{tabular}

Note: Data are presented as numbers (percentages) or means \pm standard deviations.

Abbreviations: UPS, undifferentiated pleomorphic sarcoma; ECOG PS, Eastern Cooperative Oncology Group performance status; MR, magnetic resonance imaging. 


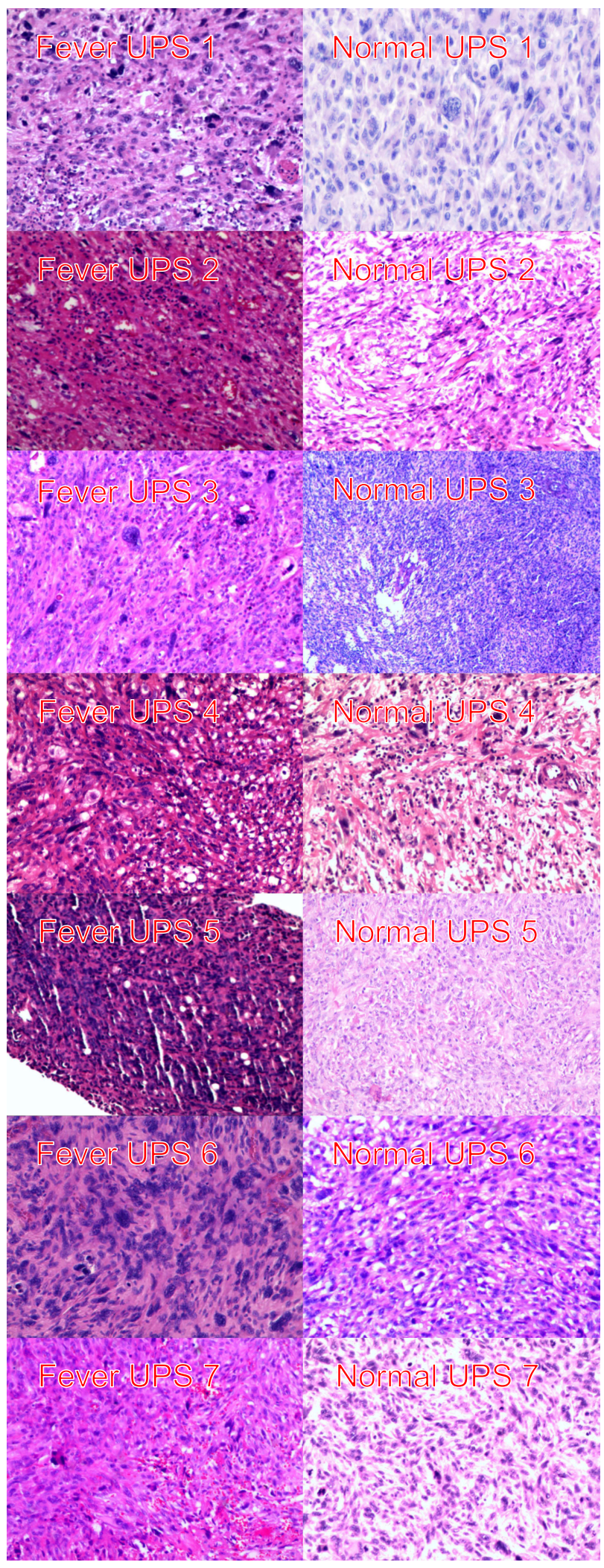

Figure I Pathological characteristics in all seven patients with UPS with fever (Fever UPS) and seven randomly selected patients with UPS without neoplastic fever (Normal UPS). Pathological manifestations were similar between the two groups. Histopathologically, all cases show high cellularity, myxoid area $<10 \%$, and pleomorphic nuclear atypia.

Abbreviation: UPS, undifferentiated pleomorphic sarcoma. 3-year survival rate than patients with UPS without neoplastic fever.

Neoplastic fever refers to continuous fever caused by the tumor itself. ${ }^{21,22}$ Previous studies have shown that only approximately $2.3 \%$ of patients with malignancies develop neoplastic fever, ${ }^{23}$ and only a small fraction of these patients have sarcoma. ${ }^{22,23}$ The cause of neoplastic fever in patients with sarcoma remains unclear. Some studies have suggested that cancer can cause fever through several mechanisms, including occult infections, inflammation, vasculitis, localized obstruction due to the tumor, and in situ production of febriferous substances by the tumor. ${ }^{17,18,24,25}$ In this study, pathological features were not significantly different between patients with UPS with and without neoplastic fever. The MRI features of patients with UPS with neoplastic fever included intratumoral necrosis and extensive soft tissue edema around the tumor. Other studies have also reported that patients with sarcoma with neoplastic fever have this characteristic appearance on MRI. ${ }^{14,18,26}$ Based on these imaging features, we speculated that the neoplastic fever was caused by internal necrosis and surrounding inflammation caused by rapid tumor growth. It is important to note that not all patients with UPS with intratumoral necrosis and peritumoral edema were found to have neoplastic fever. This suggests that two factors contribute to neoplastic fever: thermogenic factors generated by the tumor and the patient's response to these thermogenic factors. However, the specific mechanism of fever remains unclear and requires further research. In addition, the relationship between the systemic inflammatory response and the immune system in UPS patients with fever also requires further investigation, as it may uncover the mechanism by which programmed cell death protein 1 inhibitors are more effective in some UPS patients. ${ }^{27}$

Patients may be unable to tolerate neoadjuvant therapy due to neoplastic fever, which can cause discomfort symptoms and increase the treatment burden. In this study, five patients with neoplastic fever who tried to receive neoadjuvant therapy had to discontinue treatment owing to the fever. Fever symptoms in these patients led to the requirement of higher doses of antipyretics and glucocorticoids. The fever did not disappear completely until the tumor was removed. In addition, in this study, patients with fever had a higher recurrence rate. This may be due to the lack of neoadjuvant therapy, biases caused by the small number of cases, or a tendency inherent in the disease. However, due to the small number of cases, we were unable to identify the cause. However, neoplastic fever has an upside. The first 


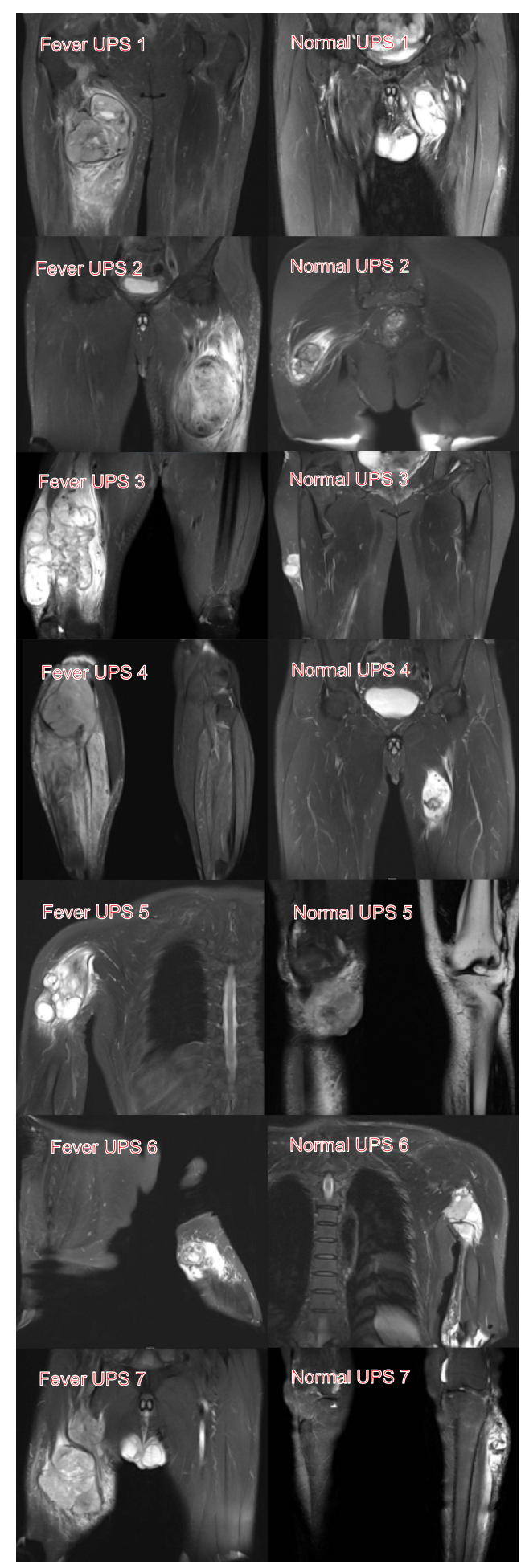

Figure 2 MRI findings of all seven patients with UPS with fever (Fever UPS) and seven randomly selected patients with UPS without neoplastic fever (Normal UPS). In patients with UPS with neoplastic fever, MRI shows necrosis inside the tumor body; all tumors span the muscular space, and extensive soft tissue edema around the tumor is noted. Most cases of UPS without neoplastic fever did not exhibit these features on MRI.

Abbreviations: MRI, magnetic resonance imaging; UPS, undifferentiated pleomorphic sarcoma. advantage is timely diagnosis. In the absence of fever, diagnosis is delayed in a significant number of patients. The existence of refractory fever of unknown cause leads the patient to visit the hospital, thereby allowing the cancer to be diagnosed in the early and middle stages. Second, according to the results of this study, concomitant neoplastic fever may be associated with lower metastasis rates.

The retrospective study design and small number of cases are the major limitations of this study. The root cause of these limitations is that UPS with neoplastic fever is an extremely rare disease. In addition, we did not measure the blood levels of systemic inflammatory cytokines and several immune molecules, such as CD3, CD8, CD20, CD34, PD-1, and PD-L1, to confirm the immune-based classification. These data can help determine whether such patients are likely to respond to immunotherapy. ${ }^{18}$ In future research, we will continue to focus on this disease.

\section{Conclusions}

UPS with neoplastic fever is a rare subtype of UPS and is characterized by intratumoral necrosis and extensive edema of the surrounding soft tissues. Patients with this subtype of UPS may have a better prognosis than those with UPS without neoplastic fever. The cause of fever in these patients necessitates further investigation.

\section{Ethics Approval and Consent to Participate}

This retrospective study was approved by the Ethics Committee of the Henan Cancer Hospital in China, and written informed consent was obtained from each patient prior to all treatment procedures. Patients were informed that their medical records were stored by the hospital and potentially used for scientific research, and that privacy would be maintained. All procedures performed in studies involving human participants were in accordance with the ethical standards of the institutional and/or national research committee and with the 1964 Helsinki declaration and its later amendments or comparable ethical standards.

\section{Acknowledgments}

The authors are grateful to all the Chinese patients and their family members for their cooperation in the study. 


\section{Author Contributions}

All authors made a significant contribution to the work reported, whether that is in the conception, study design, execution, acquisition of data, analysis and interpretation, or in all these areas; took part in drafting, revising or critically reviewing the article; gave final approval of the version to be published; have agreed on the journal to which the article has been submitted; and agree to be accountable for all aspects of the work.

\section{Funding}

There is no funding to report.

\section{Disclosure}

The authors declare they have no competing interests.

\section{References}

1. Kallen ME, Hornick JL, The 2020 WHO classification: what's new in soft tissue tumor pathology? Am J Surg Pathol. 2021;45(1):e1-e23. doi:10.1097/PAS.0000000000001552

2. Kurywchak P, Kiefer J, Lenkiewicz E, et al. Elucidating potentially significant genomic regions involved in the initiation and progression of undifferentiated pleomorphic sarcoma. Rare Tumors. 2013;5(1): e14. doi:10.4081/rt.2013.e14

3. Matushansky I, Charytonowicz E, Mills J, et al. MFH classification: differentiating undifferentiated pleomorphic sarcoma in the 21st Century. Expert Rev Anticancer Ther. 2009;9(8):1135-1144. doi:10. 1586/era.09.76

4. Pingping B, Yuhong Z, Weiqi L, et al. Incidence and Mortality of Sarcomas in Shanghai, China, During 2002-2014. Front Oncol. 2019;9:662. doi:10.3389/fonc.2019.00662

5. Lee K, Song JS, Kim JE, et al. The clinical outcomes of undifferentiated pleomorphic sarcoma (UPS): a single-centre experience of two decades with the assessment of PD-L1 expressions. Eur J Surg Oncol. 2020;46(7):1287-1293. doi:10.1016/j.ejso.2020.02.029

6. Vodanovich DA, Spelman T, May D, et al. Predicting the prognosis of undifferentiated pleomorphic soft tissue sarcoma: a 20-year experience of 266 cases. ANZ J Surg. 2019;89(9):1045-1050. doi:10.1111/ans. 15348

7. Blay JY, Le Cesne A, Demetri GD. The current reality of soft tissue sarcomas: advances, controversies, areas for improvement, and promising new treatments. Expert Rev Anticancer Ther. 2020;20 (sup1):29-39. doi:10.1080/14737140.2020.1753511

8. Spalek MJ, Kozak K, Czarnecka AM, et al. Neoadjuvant Treatment Options in Soft Tissue Sarcomas. Cancers. 2020;12(8):2061. doi:10.3390/cancers12082061

9. Gronchi A, Palmerini E, Quagliuolo V, et al. Neoadjuvant Chemotherapy in High-Risk Soft Tissue Sarcomas: final Results of a Randomized Trial from Italian (ISG), Spanish (GEIS), French (FSG), and Polish (PSG) Sarcoma Groups. J Clin Oncol. 2020;38 (19):2178-2186. doi:10.1200/JCO.19.03289

10. Bourcier K, Le Cesne A, Tselikas L, et al. Basic Knowledge in Soft Tissue Sarcoma. Cardiovasc Intervent Radiol. 2019;42(9):12 55-1261. doi:10.1007/s00270-019-02259-w
11. Tian Z, Yang Y, Yang Y, et al. High cumulative doxorubicin dose for advanced soft tissue sarcoma. BMC Cancer. 2020;20(1):1139. doi:10.1186/s12885-020-07663-x

12. Kamat NV, Million L, Yao DH, et al. The Outcome of Patients with Localized Undifferentiated Pleomorphic Sarcoma of the Lower Extremity Treated at Stanford University. Am J Clin Oncol. 2019;42(2):166-171. doi:10.1097/COC.0000000000000496

13. Sajko N, Murphy S, Tran A. Undifferentiated epithelioid sarcoma presenting as a fever of unknown origin: a case report. J Med Case Rep. 2019;13(1):24. doi:10.1186/s13256-018-1951-1

14. Doroftei NA, de Visscher N. PROLONGED FEVER WITHOUT OBVIOUS ETIOLOGY FINALLY DIAGNOSED AS HIGH-GRADE SOFT TISSUE SARCOMA. Rev Med Chir Soc Med Nat Iasi. 2016;120(1):100-104.

15. Yanagisawa R, Nakamura T, Ogiso Y, et al. Interleukin-8-producing primary cardiac undifferentiated sarcoma in a child with sustained fever. Pediatr Int. 2015;57(4):742-745. doi:10.1111/ ped. 12621

16. Katsourakis A, Noussios G, Hadjis I, et al. Primary malignant fibrous histiocytoma: a rare case. Case Rep Med. 2011;2011:134801. doi:10.1155/2011/134801

17. Osaka S, Hayakawa S, Yoshida $\mathrm{Y}$, et al. Interleukin-8 producing malignant fibrous histiocytoma with prolonged fever. Acta Histochem Cytochem. 2006;39(1):17-21. doi:10.1267/ahc.05053

18. Hashimoto $\mathrm{K}$, Nishimura $\mathrm{S}$, Ito $\mathrm{T}$, et al. Inflammatory Undifferentiated Pleomorphic Sarcoma Mimicking Bacteremia in an Elderly Patient: a Case Report. Medicina. 2021;57(2). doi:10.3390/ medicina57020175

19. Tian Z, Liu H, Zhang F, et al. Retrospective review of the activity and safety of apatinib and anlotinib in patients with advanced osteosarcoma and soft tissue sarcoma. Invest New Drugs. 2020;38 (5):1559-1569. doi:10.1007/s10637-020-00912-7

20. Weitao Y, Fangxing W, Qiqing C, et al. Efficacy and safety of apatinib in advanced sarcoma: an open-label, nonrandomized, single-center study of 45 patients. Anticancer Drugs. 2019;30(7): e0778. doi:10.1097/CAD.0000000000000778

21. Nakamura T, Matsumine A, Matsubara T, et al. Neoplastic fever in patients with bone and soft tissue sarcoma. Mol Clin Oncol. 2016;5 (5):631-634. doi:10.3892/mco.2016.1027

22. Zell JA, Chang JC. Neoplastic fever: a neglected paraneoplastic syndrome. Support Care Cancer. 2005;13(11):870-877. doi:10. 1007/s00520-005-0825-4

23. Sørensen HT, Mellemkjær L, Skriver MV, et al. Fever of unknown origin and cancer: a population-based study. Lancet Oncol. 2005;6(11):851-855. doi:10.1016/S1470-2045(05) 70346-6

24. Hirschmann JV. Fever of unknown origin in adults. Clin Infect Dis. 1997;24(3):291-300. doi:10.1093/clinids/24.3.291

25. Loizidou A, Aoun M, Klastersky J. Fever of unknown origin in cancer patients. Crit Rev Oncol Hematol. 2016;101:125-130. doi:10.1016/j.critrevonc.2016.02.015

26. Wang Z, Huang G, Yan Q, et al. Inflammatory monomorphic undifferentiated sarcoma with distinct clinical and pathological features: a 'new' entity? Pathology. 2011;43(1):48-53. doi:10.1097/PAT.0b0 $13 \mathrm{e} 328340 \mathrm{c} 1 \mathrm{f} 4$

27. Rytlewski J, Milhem MM, Monga V. Turning 'Cold' tumors 'Hot': immunotherapies in sarcoma. Ann Transl Med. 2021;9(12):1039. doi:10.21037/atm-20-6041 


\section{Publish your work in this journal}

Cancer Management and Research is an international, peer-reviewed open access journal focusing on cancer research and the optimal use of preventative and integrated treatment interventions to achieve improved outcomes, enhanced survival and quality of life for the cancer patient.

The manuscript management system is completely online and includes a very quick and fair peer-review system, which is all easy to use. Visit http://www.dovepress.com/testimonials.php to read real quotes from published authors. 